\title{
Reflecting on a Year of Elements
}

\section{by Lori Ferrins, Christine Dunne, João Borges, and Fun Man Fung}

\section{T}

he Periodic Table of Younger Chemists (PTYC) arose from a group of aspiring scientists in attendance at the 2017 World Chemistry Leadership Meeting in Sao Paulo, Brazil. This project was a celebration of the $100^{\text {th }}$ anniversary of IUPAC and the International Year of the Periodic Table. IUPAC and the International Younger Chemists Network (IYCN) joined forces to create the PTYC and honor rising stars in chemistry from around the world. Beginning in July 2018 and ending in July 2019 at the World Chemistry Congress, we unveiled and honored a diverse group of 118 outstanding younger chemists who embody the mission and core values of IUPAC. The resulting Periodic Table highlights the diversity of careers, outreach participation, and dedication to the chemistry community of those leading us into the next century of IUPAC.

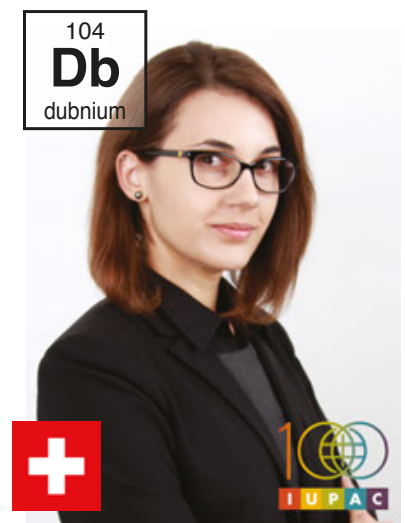

Jovana Milic Scientist, École Polytechnique Fédérale de Lausanne, Switzerland

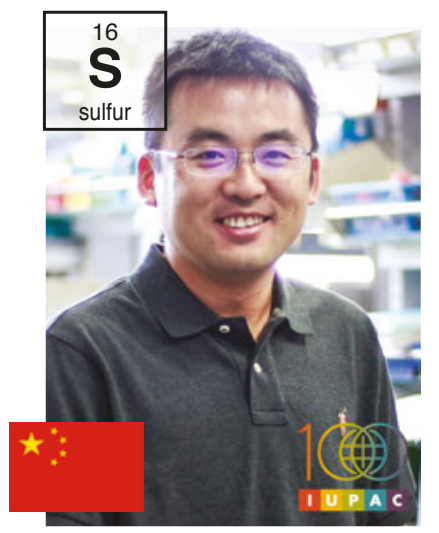

Xuefeng Jiang

Professor, East China Normal University, China
Dubnium: "This platform uniquely recognizes the contributions of young scientists beyond research excellence, including the commitment to education, international collaborations, and fostering diversity. I wish there were more initiatives that highlight these aspects in the development of young professionals as I believe that, if this was the case, we would be living in a better world!"

Sulfur: "I am proud to represent sulfur, having established 35 (Smellless, Stable and Sustainable) green sulfuration with a set of new sulfurating reagents and corresponding methodologies. I am an active contributor to IUPAC via organic chemistry achievements and outreach to the community of scholars and industry and benefited from the activities of IUPAC through academic exchange with other members in the global younger chemist community."

Each month for a year, there was immense collaboration and discussion regarding the achievements of the hundreds of nominees. To say this was a challenging task would not be giving it the true credit all the elements deserve. Everyone who was nominated was exceptional, including those who were nominated and not awarded an element. We were, and continue to be, impressed by the depth, breadth, and ambition of the younger chemists within the chemistry community.

This project was designed to open a line of communication to cross borders, tackle sustainability issues, and collaborate across labs, cultures, and professions. We are only as strong and capable as those we surround ourselves with and this group of 118 younger chemists is primed and ready to uphold the task of spearheading the development and leadership for the future of their respective chemical societies, and the one we all share together: IUPAC.

Cobalt: "I have developed long standing networks within the global chemistry community. Through these networks, I have found supportive research collaborators and funding opportunities that have served to support not only my own career but that of my students as well."

Argon: "Being selected as an element communicated that recognition and appreciation of my "little" contribution to the advancement of Chemistry. It has also catalysed my involvement with IUPAC activities. I am exploring avenues of making more impact."

Cynthia Ibeto

Senior Lecturer, University of Nigeria Nsukka, Nigeria

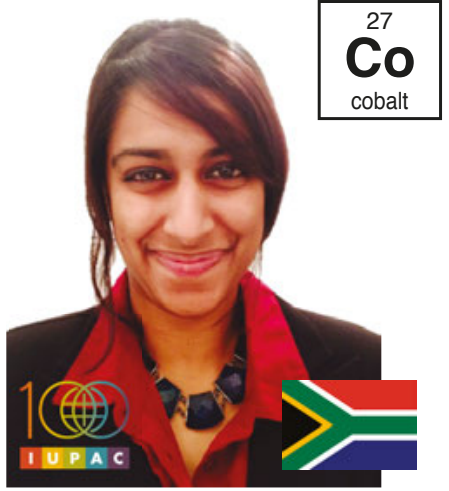

Sadhna Mathura Lecturer and Academic Coordinator, University of the Witwatersand, South Africa

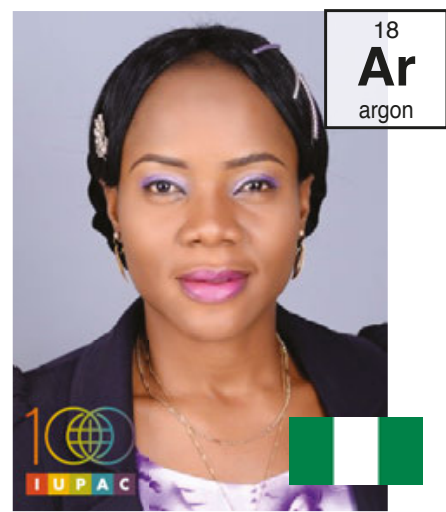




\section{A Year of Young Elements}

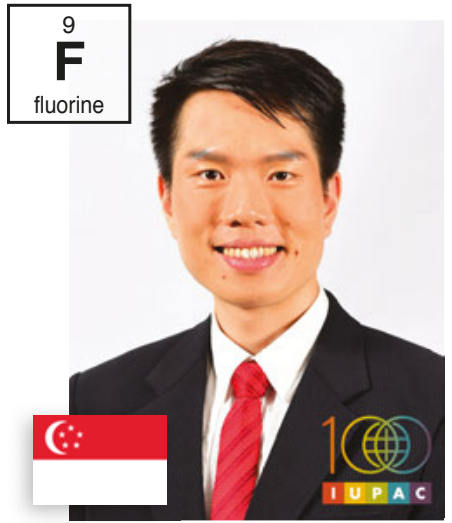

Fun Man Fung

Assistant Director (Education)

\& Chemistry Instructor, National University of Singapore

Fluorine: "I had not heard about the IYCN until I was introduced to the PTYC. I chanced upon the PTYC on Twitter when researching ways to celebrate IYPT2019 at my local university. Sensing that it was a great opportunity to promote chemistry and science, I forwarded these to my friends and colleagues in Japan and Taiwan, whom I served together with on the International Chemistry Olympiad (IChO) Steering Committee. I discovered more about the IYCN through the website and learned that I shared a similar philosophy! I was incredibly happy to learn that such a volunteering group exists that espouse mentorship for the younger chemists in the world."

The celebration of the PTYC through its collaboration with IYCN and IUPAC, was so important to highlight the strength of the young chemists around the world. We quite literally bonded over this shared connection which crossed international borders. The strong network fostered by the close-knit collaboration of IUPAC and IYCN, under this unifying project, has done an impeccable job in connecting like-minded leaders to unite and shape our futures together.

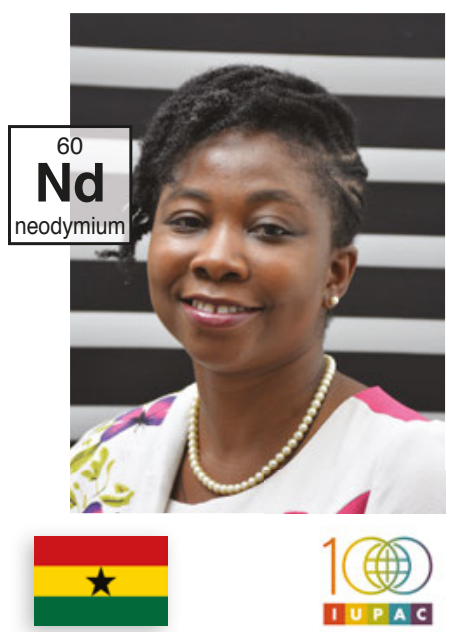

Marian Asantewa Nkansah

Senior Lecturer, Kwame

Nkrumah University of

Science \& Technology, Ghana
Neodymium: "I was in Paris for my first IUPAC Congress in 2019. Participation opened me up to the structure of IUPAC and how it runs. I was invited to join the Chemistry and Environment Division of IUPAC. As part of the process, my application to be an Affiliate of IUPAC has been accepted. I am looking forward to contributing at the Division level."

Rubidium: "My knowledge on the role and worldwide impact of
IUPAC on our daily lives' dates to my basic and high school studies in which my interest in the Chemical Sciences flourished. I became familiar with IUPAC as the world recognized scientific organization on chemical nomenclature and terminology. More recently, I wanted to increase my involvement and contribute to IUPAC's goals by addressing the 2030 United

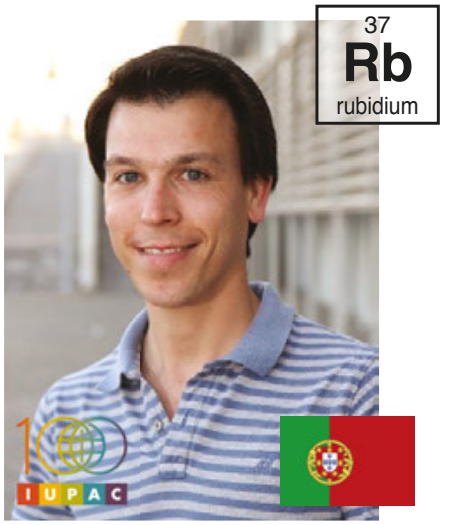

João Borges

Research Fellow,

University of Aveiro, Portugal Nations Sustainable Development Goals, as well as by sharing my passion and spreading scientific knowledge on the interdisciplinary field of chemistry and its impact via science outreach and public engagement activities.

In 2018, when I heard about the IYCN, which is an affiliated organization of IUPAC that connects, supports and advocates for chemists who are in the early stage of their career, I learnt different ways to contribute towards fulfilling the IUPAC goals. In fact, I promptly identified myself with the vision, mission, aims, projects, and activities launched by IYCN which led me to expressing my willingness to join the growing network.

I became familiar with the IUPAC100 PTYC launched by IUPAC and IYCN, in celebration of the $100^{\text {th }}$ anniversary of IUPAC and the 2019 International Year of the Periodic Table and was selected as the representative of the element Rubidium for embodying the mission and core values of IUPAC. Such recognition enabled me to connect with, and learn from, many younger chemists' element awardees from across the globe at the 47 th IUPAC World Chemistry Congress (47th IUPAC WCC). This was my first ever IUPAC WCC, which allowed me to significantly strengthen my bonds with IUPAC, proudly acting as a "young ambassador" of the organization and contributing to aid in fulfilling its noble goals and shaping the future of chemistry worldwide.

IUPAC2019 also featured the IYCN General Assembly in which I was honored to represent Portugal as a delegate and had the opportunity to actively and effectively contribute to redefine the mission and vision of IYCN. The IYCN General Assembly was a very enriching meeting in which I was fortunate to meet, interact, and learn from many younger chemists from countries worldwide. The General Assembly also raised my willingness and desire to keep on fighting (and volunteering) toward supporting, connecting, advocating, 
and empowering young chemists to progress and fulfill their career goals.

Everyone has a voice within IUPAC, and we should all contribute toward its shared mission with IYCN by aiding in the development of a common language for chemistry."
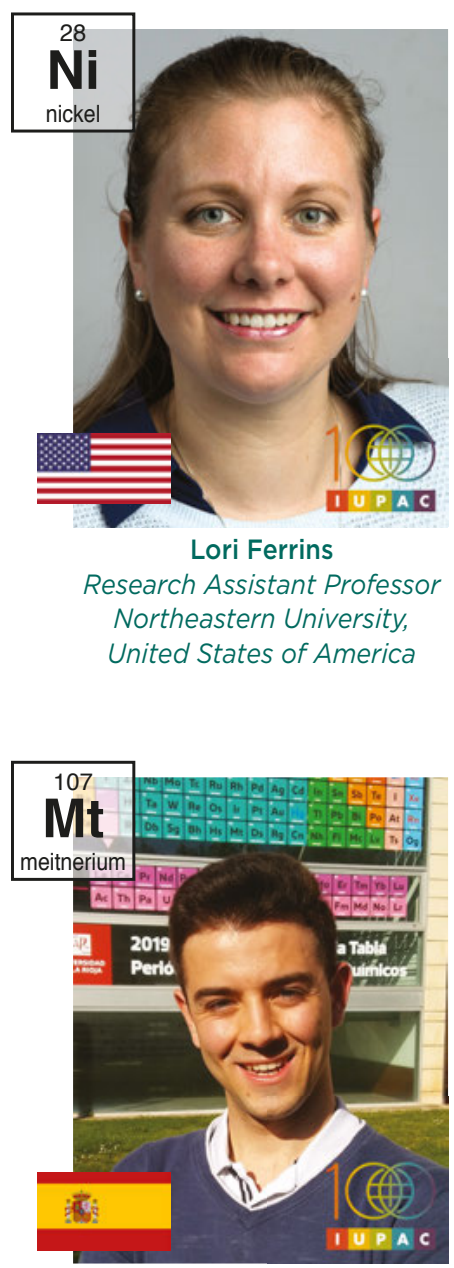

Victor Sabanza

Undergraduate Student, University of La Rioja, Spain

This project is just the beginning of not only empowering younger chemists within the IUPAC community but also a continued collaboration between IUPAC and IYCN. The bonds made throughout 2019 will continue to grow and we intend to nurture this network of outstanding younger chemists with several initiatives.

Meitnerium: "As an undergraduate student, being here is an outstanding honour. However, the most impressive fact is that I was selected for sharing my passion for Chemistry and the Periodic Table with younger students. I believe inspiring other people and being inspired by others is key to science."
We are working to develop a webinar series, featuring presentations from the awardees of the periodic table. We want to give the outstanding younger chemists an opportunity to talk about their research and contributions to the chemical science community. This series would then culminate in a face-to-face meeting at the IUPAC congress in 2021 (iupac2021.org/).

The collaboration and in-person leadership development at the IUPAC World Chemistry Congresses will continue to be cultivated in the years to come. The IUPAC 2021 WCC in Montreal, Canada will be the first opportunity for the legacy of the PTYC to continue. We hope to provide a platform in which the awardees can share their research, career developments, and future outlook for the IUPAC community. By continuing to honor these outstanding chemists we will be able to show how the power of a diverse community, in collaboration with IYCN, can impact not only IUPAC, but also the greater scientific community. Our crystals are just beginning to form, and we are so excited to see them grow. In April 2020, a new IUPAC project highlighting the activity and engagement of the Periodic Table of Younger Chemist awardees with the IYCN and IUPAC has been initiated See project 2020-012-2-020 (https://iupac.org/project/2020-012-2-020) for details and updates. Lead: "There are many talented young chemists in the world, and to be selected as one of the 118 elements was an honor; we are a symbol of the diversity of careers globally. I see the PTYC as an opportunity to keep in touch for future projects and collaborations and I am curious to see where our paths might cross again."

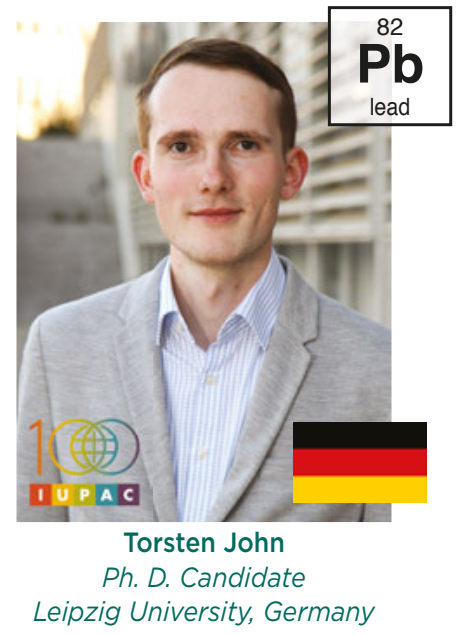

Congratulations to all the elements, and welcome to the IUPAC family!

https://iupac.org/100/pt-of-chemist/ 